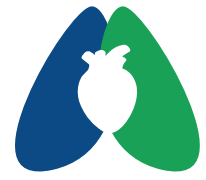

ASSOBRAFIR C I Ê N C I A

\section{Há boa correlação entre os desfechos clínicos e a qualidade do sono em indivíduos com DPOC?a}

\author{
Is there good correlation between clinical outcomes and sleep \\ quality in individuals with COPD?
}

Roberta Bordignon Rodrigues Machado ${ }^{1}$; ; Letícia Fernandes Belo ${ }^{1}$ (1);

Daniele Caroline Dala Pola (1D; Raquel Pastrello Hirata' ${ }^{1}$; ; Nidia Aparecida Hernandes ${ }^{1}$ (D); Fabio Pitta ${ }^{1 *}$ (1)

\begin{abstract}
Resumo
Introdução: Não está claro se a pior qualidade do sono apresentada por indivíduos com Doença Pulmonar Obstrutiva Crônica (DPOC) correlaciona-se aos sintomas comumente desencadeados pela doença. Objetivos: Investigar se existe correlação entre a qualidade do sono com estado funcional, estado de saúde, dispneia, ansiedade, depressão e qualidade de vida em indivíduos com DPOC. Métodos: Indivíduos com DPOC foram avaliados transversalmente quanto à qualidade do sono pelo Pittsburgh Sleep Quality Index (PSQI) e esta foi correlacionada com os instrumentos: Medical Research Council scale (MRC), Hospital Anxiety and Depression scale (HADS), London Chest Activity of Daily Living (LCADL), Chronic Respiratory Questionnaire (CRQ) e COPD Assessment Test (CAT). Os escores gerais e por domínios foram utilizados para as análises. As correlações foram avaliadas pelo coeficiente de correlação de Spearman, e a significância estatística de $P<0,05$. Resultados: Foram incluídos dezoito indivíduos com DPOC (67\% homens; 64[60-71] anos; VEF, 48[41-58]\%predito), em sua maioria com má qualidade do sono (6[4-9]pontos). A qualidade do sono e a disfunção diurna (PSQI) correlacionaram-se moderadamente com os domínios fadiga e emocional do CRQ $(-0,68 \leq$ $r \leq 0,61)$ e fortemente aos distúrbios do sono, assim como com o sono avaliado pelo CAT $(r=-0,80 ;-0,79$ e 0,73 , respectivamente). Além disso, a latência do sono correlacionou-se com o domínio auto-controle do CRQ $(r=-0,70)$ e com a dispneia do CAT $(r=0,47)$. Conclusão: A qualidade do sono dos indivíduos com DPOC correlaciona-se moderadamente a fortemente com a fadiga, questões emocionais, dispneia, qualidade de vida e funcionalidade dessa população.
\end{abstract}

Apresentação dos dados em evento: Congresso dos 40 anos de Fisioterapia da UEL: história, evolução e desafios. 20 anos da residência em Fisioterapia da UEL. 10 anos do programa de pós-graduação Stricto Sensu em Ciências da Reabilitação (2019); e X SULBRAFIR - Congresso SulBrasileiro de Fisioterapia Respiratória, Cardiovascular e em Terapia Intensiva (2019).

'Laboratório de Pesquisa em Fisioterapia Pulmonar (LFIP), Universidade Estadual de Londrina (UEL), Londrina, PR, Brasil

Como citar: Machado RBR, Belo LF, Pola DCD, Hirata RP, Hernandes NA, Pitta F. Há boa correlação entre os desfechos clínicos e a qualidade do sono em indivíduos com DPOC?. ASSOBRAFIR Ciênc. 2022;13:e44615. https://doi.org/10.47066/21779333. AC.2020.0039

Submissão em: Setembro 27, 2021 Aceito em: Dezembro 15, 2021

Estudo realizado em: Laboratório de Pesquisa em Fisioterapia Pulmonar (LFIP), Universidade Estadual de Londrina (UEL), Londrina, PR, Brasil.

Aprovação ética: CAAE:

57961716.2.0000.5231 da Universidade Estadual de Londrina, $\mathrm{n}^{\circ} 2.469 .386$.

*Autor correspondente: Fabio Pitta. E-mail: fabiopitta@uol.com.br
Palavras-chave: Doença Pulmonar Obstrutiva Crônica; Sono; Qualidade de vida; Fadiga; Dispneia.

\begin{abstract}
Background: It is not clear whether a poor quality of sleep associated with Chronic Obstructive Pulmonary Disease (COPD) correlates with the symptoms commonly triggered by the disease. Aim: To investigate whether there are correlation among the quality of sleep and functional status, health status, dyspnea, anxiety, depression and quality of life in individuals with COPD. Methods: Individuals with CPOD were cross-sectionally assessed for sleep quality by the Pittsburgh Sleep Quality Index [PSQI] and this was correlated with the following instruments: Medical Research Council scale (MRC), Hospital Anxiety and Depression scale (HADS), London Chest Activity of Daily Living (LCADL), Chronic Respiratory Questionnaire (CRQ) and COPD Assessment Test (CAT). The total and domain scores were used for the analyzes. Correlations were assessed using Spearman's correlation coefficient, and the statistical significance of $P<0.05$. Results: Eighteen individuals with COPD were included (67\% men; 64 [60-71] years; FEV 48 [41-58]\%predicted), mostly characterized by poor sleep quality (6[4-9]). Sleep quality and daytime dysfunction (PSQI) were correlated moderately with the fatigue and emotional domains of the CRQ $(-0.68 \leq r \leq 0.61)$ and strongly correlated with sleep disorders, as well as sleep assessed by the CAT ( $r=-0.80 ;-0.79$ and 0.73 , respectively). In addition, sleep latency was correlated with the CRQ self-control domain $(r=-0.70)$ and with CAT dyspnea $(r=0.47)$. Conclusion: Quality of sleep of individuals with COPD correlates moderately-to-strongly with fatigue, emotional issues, dyspnea, quality of life and functionality in this population.
\end{abstract}

Keywords: Chronic Obstructive Pulmonary Disease; Sleep; Quality of Life; Fatigue; Dyspnea.

Este é um artigo publicado em acesso aberto (Open Access) e distribuído sob a licença Creative Commons Attribution NonComercial ShareAlike License, que permite uso, distribuição e reprodução em qualquer meio, sem restrições desde que sem fins comerciais e que o trabalho original seja corretamente citado e de forma que não indique endosso ao trabalho feito. Adicionalmente, qualquer trabalho derivado deverá ser publicado sob a mesma licença. 


\section{INTRODUÇÃO}

A doença pulmonar obstrutiva crônica (DPOC) é uma doença crônica, prevenível e tratável caracterizada pela obstrução progressiva e persistente ao fluxo aéreo ${ }^{1}$. Apesar do diagnóstico ser dado pela prova de função pulmonar, além da dispneia e dos efeitos pulmonares, a DPOC cursa com efeitos extrapulmonares significativos tais como, disfunção muscular periférica, descondicionamento físico, diminuição da qualidade de vida e sintomas de ansiedade e depressão, entre outros ${ }^{2,3}$. Essas alterações acima mencionadas podem interferir diretamente na qualidade do sono.

Existem alguns fatores de que podem acentuar os sintomas da DPOC e interferir na noite de sono dessa população, como a tosse por exemplo. Shorofsky e colaboradores demonstraram como resultado de um estudo coorte com uma ampla amostra, que os sintomas dos indivíduos com DPOC, como tosse e chiado, interferem na duração total do sono e/ou fazem com que estes indivíduos demorem mais para dormir predispondo à sonolência diurna, alterações cognitivas, imunológicas e maior risco de desenvolvimento de exacerbações agudas da doença ${ }^{4,5}$. Além disso, Nunes e colaboradores evidenciaram que $70 \%$ dos sujeitos com DPOC moderada a muito grave, foram identificados objetivamente com uma má qualidade do sono, e isso se mostrou como um fator determinante na qualidade de vida desses indivíduos ${ }^{6}$. Tendo em vista os fatores acima mencionados, uma boa avaliação da qualidade do sono em indivíduos com DPOC se faz necessária visando entender melhor o impacto da qualidade do sono nessa população.

Embora já se saiba que os indivíduos com diagnóstico de DPOC apresentem alterações na qualidade do sono ${ }^{4}$, ainda não está claro se os sintomas mais comuns desencadeados pela doença apresentam relação com a qualidade do sono desses indivíduos. Por isso, o objetivo do presente estudo foi investigar se existe correlação entre a qualidade do sono com estado funcional, estado de saúde, dispneia, ansiedade, depressão e qualidade de vida em indivíduos com DPOC. Partiu-se da hipótese que há correlação ao menos moderada entre a qualidade do sono e os outros desfechos.

\section{MÉTODOS}

Trata-se de um estudo transversal, com uma amostra de conveniência composta por indivíduos com diagnóstico de DPOC, segundo critérios da Global Initiative for Obstructive Lung Disease (GOLD)'. Os indivíduos foram recrutados durante a avaliação inicial para participação em um programa de treinamento físico no ambulatório de fisioterapia respiratória do Hospital Universitário da Universidade Estadual de Londrina. Após o esclarecimento dos procedimentos e dos objetivos do estudo, e após concordarem com a sua participação, todos indivíduos assinaram um termo de consentimento livre e esclarecido. A pesquisa foi aprovada pelo Comitê de Ética em Pesquisa da instituição. Aprovação ética: CAAE: 57961716.2.0000.5231 da Universidade Estadual de Londrina, $\mathrm{n}^{\circ} 2.469 .386$.

Foram incluídos indivíduos com DPOC ${ }^{1}$ estável, ou seja, sem exacerbações agudas da doença ou infecções respiratórias no último mês; sem comorbidades que pudessem influenciar na realização dos testes; e que não tivessem participado de programa de treinamento físico no último ano. E foram excluídos os indivíduos que apresentaram incapacidade de concluir o protocolo proposto por algum motivo (p.ex.: questões pessoais ou de saúde, dificuldade com horários etc.)

Os indivíduos foram avaliados quanto à função pulmonar, qualidade do sono, sintomas de ansiedade e depressão, estado funcional, dispneia na vida diária, qualidade de vida e estado de saúde, conforme descrito a seguir. As avaliações ocorreram em no máximo três dias, sendo que todos os questionários foram aplicados por meio de entrevista e em sequência padronizada, por um avaliador treinado. Apenas o questionário de qualidade do sono foi aplicado por um avaliador distinto, também treinado.

\section{Função pulmonar}

A espirometria foi realizada de acordo com a padronização internacional ${ }^{7}$ por meio do pletismógrafo (Vmax ${ }^{\circledR}$ Carefusion, Germany). Os valores de referência utilizados foram os desenvolvidos para a população brasileira ${ }^{8}$.

\section{Qualidade do sono}

A qualidade do sono foi avaliada pelo Pittsburgh Sleep Quality Index (PSQI), desenvolvido por Buysse e colaboradores, e validado no Brasil por Bertolazzi e colaboradores ${ }^{9,10}$. O PSQI avalia a qualidade e as perturbações durante o sono no período de um mês. O instrumento é composto por 19 questões direcionadas ao indivíduo avaliado e cinco questões dirigidas ao cônjuge ou acompanhante de quarto. As primeiras 19 questões são divididas em sete componentes, sendo estes: C1 qualidade subjetiva do sono, C2 - latência do sono, C3 duração do sono, C4 - eficiência habitual do sono, C5 distúrbios do sono, C6 - uso de medicação para dormir e C7 - disfunção diurna.

As cinco últimas questões são utilizadas apenas para a análise clínica e não contribuem para a pontuação total do índice. Cada componente é graduado de 0-3 pontos (nenhuma dificuldade - dificuldade grave) e da soma deles se dá o escore total, que varia de 0 a 21 pontos. Quanto maior a pontuação pior é a qualidade do sono e pontuações maiores que 5 indicam uma má qualidade do sono $0^{9,10}$. 


\section{Sintomas de ansiedade e depressão}

Para avaliar os sintomas de ansiedade e depressão foi utilizada a Hospital Anxiety and Depression scale (HADS). Validada previamente em língua portuguesa, essa escala consiste em 14 questões subdividida em duas subescalas, sendo sete questões para ansiedade e sete para depressão. Os escores variam de 0 a 21 pontos para cada subescala. Pontuações de 9 pontos ou mais indicam a presença de sintomas de ansiedade ou depressão ${ }^{11,12}$

\section{Estado funcional}

O estado funcional foi avaliado pela escala London Chest Activity of Daily Living (LCADL), validada previamente para a língua portuguesa ${ }^{13,14}$. Essa escala possui 15 itens de atividades de vida diária (AVDs), divididos em quatro domínios: cuidado pessoal (4 itens), cuidado doméstico (6 itens), atividade física ( 2 itens) e lazer ( 3 itens), pontuados de 0-5 cada. O escore total varia de 0 a 75 pontos e valores mais altos na escala indicam maior limitação nas AVDs.

\section{Dispneia}

O impacto da dispneia na vida diária foi avaliado por meio da escala Medical Research Council (MRC), validada para uso no Brasil ${ }^{15}$. A escala é composta por 5 itens e o paciente escolhe o item que melhor corresponder ao seu grau de limitação na vida diária ${ }^{15}$.

\section{Qualidade de vida}

Para mensurar a qualidade de vida foi utilizado o Chronic Respiratory Questionnaire (CRQ), também validado para língua portuguesa ${ }^{16}$. O CRQ conta com 20 questões, divididas em quatro domínios: dispneia (5 questões), fadiga (4 questões), função emocional (7 questões) e autocontrole (4 questões). Quanto maior a pontuação, melhor a qualidade de vida ${ }^{16}$.

\section{Estado de saúde}

O estado de saúde foi avaliado pelo COPD Assessment Test (CAT), já validado para uso no Brasili ${ }^{17}$. Esse instrumento é composto por 8 itens (tosse, secreção, aperto no peito, falta de ar, limitações nas atividades domésticas, confiança em sair de casa, sono e energia), pontuados de 0 a 5 pontos. O escore total varia de 0 a 40 pontos e valores mais altos indicam maior impacto clínico da DPOC.

\section{Análise estatística}

Os softwares utilizados para a análise estatística foram o SPSS Statistical Package (IBM SPSS Statistics, Chicago, IL, EUA) e o GraphPad Prism 6.0 (GraphPad Software, San Diego, CA, EUA). A análise não-paramétrica foi utilizada pelo tamanho de amostra e pelo fato de se tratar da análise de dados ordinais. Sendo assim, os dados foram descritos em mediana [intervalo interquartílico 25-75\%].
O coeficiente de correlação de Spearman foi utilizado para verificar a correlação da qualidade de sono com os sintomas e o estado funcional nos indivíduos com DPOC. A interpretação das correlações foi como se segue: fraca $0<r \leq 0,30$; moderada 0,30<r $\leq 0,60$; forte $0,60<r \leq$ 0,90; muito forte $0,90<r<1^{18}$. A significância estatística adotada foi de $P<0,05$. Utilizou-se o Software $G *$ Power 3.1.5 para realização do cálculo do poder da amostra, que demonstrou um poder de 0,80 para as correlações $\geq 0,60$.

\section{RESULTADOS}

Foram inclusos no presente estudo 18 indivíduos com diagnóstico de DPOC moderada à grave (64 [60-71] anos, 67\% homens, VEF 48 [41-58] \%pred). Uma participante foi excluída das análises por apresentar dados fora dos padrões da amostra (outlier). Os dados demográficos da amostra estão descritos na Tabela 1.

A Tabela 2 contém a descrição da qualidade do sono, dispneia, qualidade de vida, estado de saúde e funcionalidade dos indíviduos estudados. De forma geral os participantes do estudo apresentaram má qualidade de sono, com um escore mediano de 6 pontos no PSQI, moderada limitação pela dispneia nas atividades do diaa-dia segundo os resultados da MRC, e dos domínios de dispneia do CRQ e do CAT.

Os resultados do PSQI (C1, C7 e total) correlacionaram-se fortemente e negativamente com os domínios fadiga e emocional do CRQ $(-0,68 \leq r \leq 0,61)$ (Tabela 3 e Figuras 1 e 2). A latência do sono (C2), ou seja, o período que é preciso para realizar a transição da vigília para o sono total, correlacionou-se fortemente com o domínio auto-controle do CRQ $(r=-0,70)$. Os distúrbios do sono (C5), foram associados a maiores sintomas de fadiga ( $r=-$ $0,80)$, emocional $(r=-0,79)$ e sono $(r=0,73)$ (Figura 3). Já a disfunção diurna (C7), correlacionou-se com os sintomas emocionais $(r=-0,49)$. Os distúrbios do sono (C5) foram

Tabela 1. Características demográficas da amostra.

\begin{tabular}{|c|c|}
\hline Variáveis & $n=18$ \\
\hline Sexo (n, (\%) homens) & $12,(67)$ \\
\hline Idade (anos) & $64[60-71]$ \\
\hline IMC $\left(\mathrm{Kg} / \mathrm{m}^{2}\right)$ & $30[27-32]$ \\
\hline GOLD I/II/III/IV (\%) & 0/50/39/11 \\
\hline $\operatorname{VEF}_{1}(L)$ & $1,40[1,00-1,71]$ \\
\hline VEF $_{1}$ (\%predito) & $48[41-58]$ \\
\hline CVF (L) & $2,52[1,96-3,67]$ \\
\hline CVF (\%predito) & 72 [62-96] \\
\hline $\mathrm{VEF}_{1} / \mathrm{CVF}(\%)$ & 51 [40-63] \\
\hline
\end{tabular}

IMC: Índice de massa corpórea; GOLD: Global initiative for chronic obstructive lung disease; $V_{E F}$ : Volume expiratório forçado no primeiro segundo; CVF: Capacidade vital forçada. 
Tabela 2. Características de qualidade do sono (PSQI), qualidade de vida (CRQ), dispneia (MRC), sintomas de ansiedade e depressão (HADS), estado de saúde (CAT) e estado funcional (LCADL) nos indivíduos com DPOC.

\begin{tabular}{|c|c|c|c|}
\hline Instrumentos & Mediana [IIQ 25-75\%] & Instrumentos & Mediana [IIQ 25-75\%] \\
\hline \multicolumn{2}{|c|}{ PSQI, pontos } & HADS-A, pontos & $3[1-5]$ \\
\hline C1 - Qualidade do sono & $1[1-2]$ & HADS-D, pontos & $4[2-6]$ \\
\hline C2 - Latência do sono & $1[0-2]$ & \multicolumn{2}{|c|}{ CRQ, pontos } \\
\hline C3 - Duração do sono & $1[0-2]$ & Dispneia & $4[3-5]$ \\
\hline C4 - Eficiência do sono & $0[0-2]$ & Fadiga & $5[4-6]$ \\
\hline C5 - Distúrbios do sono & $1[1-2]$ & Emocional & $6[4-6]$ \\
\hline C6 - Medicação para dormir & $0[0-1]$ & Auto-controle & $7[6-7]$ \\
\hline C7 - Disfunção diurna & $1[0-1]$ & \multicolumn{2}{|c|}{ CAT, pontos } \\
\hline Total & 6 [4-9] & Tosse & $1[0-3]$ \\
\hline \multicolumn{2}{|c|}{ LCADL, pontos } & Secreção & $1[0-2]$ \\
\hline Cuidado pessoal & $5[4-8]$ & Pressão no peito & $0[0-1]$ \\
\hline Doméstico & $8[4-11]$ & Falta de ar & $5[4-5]$ \\
\hline Atividade física & $4[3-5]$ & Limitação AVDs & $2[1-3]$ \\
\hline Lazer & $3[3-5]$ & Confiança & $0[0-3]$ \\
\hline Total & $22[16-27]$ & Sono & $0[0-3]$ \\
\hline \multirow[t]{2}{*}{ MRC, pontos } & $3[2-3]$ & Energia & $3[0-3]$ \\
\hline & & Total & $15[8-20]$ \\
\hline
\end{tabular}

PSQI: Pittsburgh Sleep Quality Index; C1: Qualidade subjetiva do sono; C2: Latência do sono; C3 Duração do sono; C4: Eficiência do sono; C5: Distúrbios do sono; C6: Uso de medicação para dormir; C7: Disfunção diurna; CRQ: Chronic Respiratory Questionnaire; MRC: Medical Research Council; HADS-A: Hospital Anxiety and Depression Scale - subescala ansiedade; HADS-D: Hospital Anxiety and Depression Scale - subescala depressão; CAT: COPD Assessment Test; AVDs: atividades de vida diária; LCADL: London Chest Activity of Daily Living.

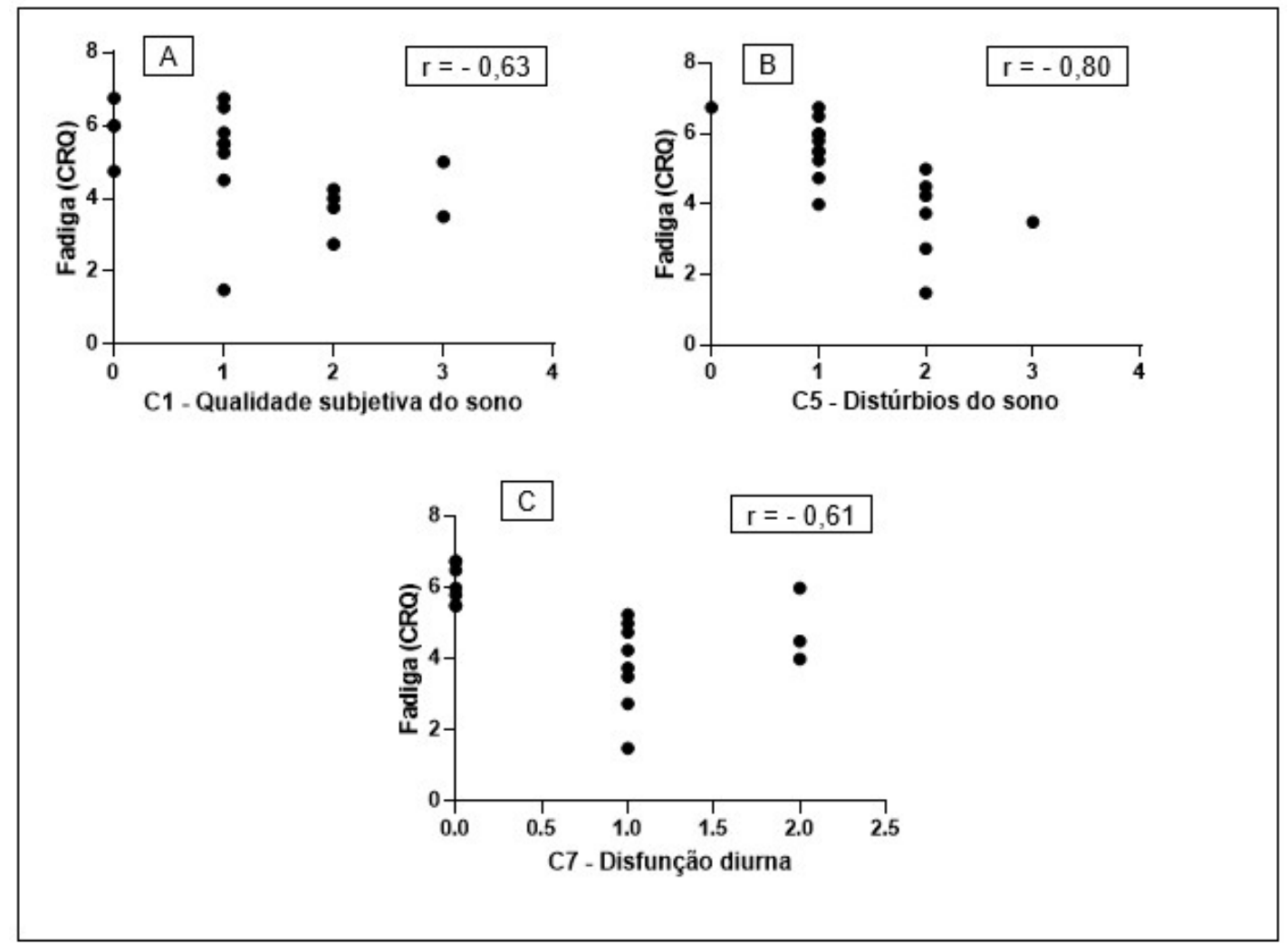

Figura 1. Correlação da fadiga com a qualidade subjetiva do sono (A); com os distúrbios do sono (B) e com a disfunção diurna (C). 
Tabela 3. Correlações da qualidade de vida, sintomas de dispneia, ansiedade e depressão, estado de saúde e estado funcional com a qualidade do sono nos indivíduos com DPOC.

\begin{tabular}{|c|c|c|c|c|c|c|c|c|}
\hline & \multicolumn{8}{|c|}{ Componentes PSQI } \\
\hline & \multirow{2}{*}{$\begin{array}{c}\text { C1 } \\
\text { Qualidade } \\
\text { do sono }\end{array}$} & $\mathrm{C} 2$ & $\mathrm{C} 3$ & \multirow{2}{*}{$\begin{array}{c}\text { C4 } \\
\text { Eficiência } \\
\text { do sono }\end{array}$} & \multirow{2}{*}{$\begin{array}{c}\text { C5 } \\
\begin{array}{c}\text { Distúrbios } \\
\text { do sono }\end{array}\end{array}$} & \multirow{2}{*}{$\begin{array}{c}\text { C6 } \\
\text { Medicação } \\
\text { para dormir }\end{array}$} & \multirow{2}{*}{$\begin{array}{c}\text { C7 } \\
\text { Disfunção } \\
\text { diurna }\end{array}$} & \multirow[b]{2}{*}{ Total } \\
\hline & & $\begin{array}{l}\text { Latência do } \\
\text { sono }\end{array}$ & $\begin{array}{l}\text { Duração do } \\
\text { sono }\end{array}$ & & & & & \\
\hline \multicolumn{9}{|l|}{ CRQ } \\
\hline Dispneia & $-0,12$ & $-0,26$ & $-0,11$ & $-0,06$ & $-0,22$ & 0,14 & $-0,19$ & $-0,35$ \\
\hline Fadiga & $-0,63^{* *}$ & $-0,24$ & $-0,05$ & 0,12 & $-0,80 * *$ & 0,14 & $-0,61 * *$ & $-0,55^{*}$ \\
\hline Emocional & $-0,67 * *$ & $-0,3$ & $-0,2$ & $-0,04$ & $-0,79 * *$ & 0,05 & $-0,49^{*}$ & $-0,68 * *$ \\
\hline $\begin{array}{c}\text { Auto- } \\
\text { controle }\end{array}$ & $-0,15$ & $-0,7$ & 0,14 & 0,19 & $-0,46$ & $-0,22$ & $-0,38$ & $-0,19$ \\
\hline MRC & $-0,16$ & 0,9 & $-0,48 *$ & $-0,45$ & $-0,17$ & 0,6 & $-0,14$ & $-0,32$ \\
\hline HADS-A & 0,32 & 0,22 & $-0,01$ & $-0,03$ & $0,52^{*}$ & 0,04 & 0,42 & 0,37 \\
\hline HADS-D & $-0,26$ & $-0,12$ & $-0,41$ & $-0,1$ & 0,3 & $-0,18$ & 0,46 & $-0,23$ \\
\hline \multicolumn{9}{|l|}{ CAT } \\
\hline Tosse & 0,1 & $-0,2$ & 0,12 & 0,24 & 0,3 & $-0,13$ & $-0,21$ & 0,12 \\
\hline Secreção & 0,19 & $-0,31$ & 0,13 & 0,11 & 0,41 & $-0,03$ & 0,3 & 0,12 \\
\hline $\begin{array}{l}\text { Pres. no } \\
\text { peito }\end{array}$ & 0,2 & 0,04 & $-0,08$ & 0,11 & $0,52 *$ & $-0,33$ & 0,26 & 0,12 \\
\hline Falta de ar & $-0,13$ & $0,47^{\star}$ & $-0,16$ & $-0,08$ & $-0,16$ & $-0,05$ & 0,2 & 0,12 \\
\hline Limit. AVDs & $-0,19$ & 0,07 & $-0,29$ & $-0,32$ & 0,21 & $-0,03$ & 0,32 & $-0,08$ \\
\hline Confiança & $-0,25$ & 0,14 & $-0,28$ & $-0,39$ & $-0,16$ & 0,36 & 0,32 & $-0,04$ \\
\hline Sono & 0,42 & 0,12 & $-0,36$ & $-0,28$ & $0,73 * *$ & $-0,2$ & 0,29 & 0,22 \\
\hline Energia & 0,19 & $-0,23$ & $-0,08$ & $-0,46$ & 0,32 & 0,46 & 0,35 & 0,14 \\
\hline Total & 0,14 & 0,01 & $-0,29$ & $-0,33$ & $0,5^{*}$ & 0,06 & 0,46 & 0,14 \\
\hline \multicolumn{9}{|l|}{ LCADL } \\
\hline $\begin{array}{l}\text { Cuid. } \\
\text { pessoal }\end{array}$ & $-0,17$ & 0,31 & $-0,18$ & $-0,02$ & 0,23 & 0,16 & 0,44 & 0,19 \\
\hline Doméstico & $-0,01$ & 0,26 & $-0,3$ & $-0,19$ & 0,33 & 0,1 & 0,4 & 0,18 \\
\hline Ativ. física & $-0,26$ & 0,33 & $-0,25$ & 0,13 & 0,06 & $-0,22$ & 0,28 & 0,03 \\
\hline Lazer & 0,09 & 0,24 & $-0,17$ & $-0,02$ & $0,5^{*}$ & $-0,08$ & 0,37 & 0,25 \\
\hline Total & 0 & 0,37 & $-0,31$ & $-0,16$ & 0,4 & 0,01 & 0,5 & 0,23 \\
\hline
\end{tabular}

PSQI: Pittsburgh Sleep Quality Index; CRQ: Chronic Respiratory Questionnaire; MRC: Medical Research Council; HADS-A: Hospital Anxiety and Depression Scale - subescala ansiedade; HADS-D: Hospital Anxiety and Depression Scale - subescala depressão; CAT: COPD Assessment Test; AVDs: atividades de vida diária; LCADL: London Chest Activity of Daily Living. * $\mathrm{p}<0,05 ;{ }^{*} \mathrm{p}<0,01$.

associados moderadamente aos maiores sintomas de ansiedade (HADS-A) $(r=0,52)$ e pressão no peito $(r=0,52)$. Além disso, a queixa de falta de ar (avaliada pelo CAT) demontrou estar relacionada ao tempo que os indivíduos com DPOC demoram para dormir, com uma correlação de $r=0,47$ com o C2 do PSQI. As demais correlações entre os desfechos dos questionário, com a qualidade do sono nos indivíduos da amostra estão descritas na Tabela 3.

\section{DISCUSSÃO}

O presente estudo investigou a correlação entre a qualidade do sono de indivíduos com DPOC com seu estado funcional e seus sintomas de dispneia, fadiga, ansiedade, depressão e qualidade de vida. De modo geral as principais queixas relacionadas a doença foram os sintomas de fadiga, as questões emocionais e a dispneia. Os componentes do PSQI que melhor correlacionaram-se 




Figura 2. Correlações do domínio emocional do CRQ com a pontuação total e os componentes 1 e 5 do PSQI (paineis A, B e C respectivamente).

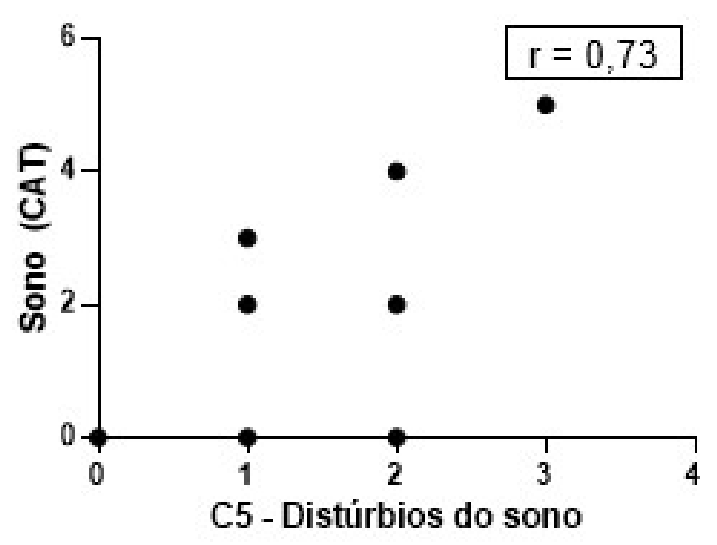

Figura 3. Correlação entre a avaliação do sono pelo COPD Assessment Test e dos distúrbios do sono avaliados pelo Pittsburgh Sleep Quality Index.

com os desfechos analisados foram qualidade subjetiva do sono (C1), distúrbios do sono (C5), disfunção diurna (C7) e a pontuação total do PSQI.

Oliveira e colaboradores mostraram previamente que mais de $70 \%$ dos indivíduos de uma amostra de 39 indivíduos com DPOC apresentam queixas de fadiga ${ }^{19}$. No entanto, apesar de ser considerado um sintoma frequente na DPOC, a fadiga tem recebido relativamente pouca atenção quando comparada a outras manifestações sistêmicas da doença. O presente estudo encontrou correlação forte da fadiga com a má qualidade do sono por meio dos componentes do PSQI qualidade subjetiva do sono (C1), distúrbios do sono (C5) e disfunção diurna (C7). Isso demonstra a relevância da avaliação da fadiga, mesmo que de forma autorrelatada. Um estudo prévio também encontrou relação da fadiga e do sono em 
adultos com doenças obstrutivas, sendo eles 12,8\% DPOC, corroborando dessa forma com os presentes achados ${ }^{20}$.

As questões emocionais, isto é, a maneira como o indivíduo enfrenta a doença e o seu dia a dia, também se correlacionaram fortemente à qualidade do sono por meio dos componentes qualidade subjetiva do sono (C1), distúrbios do sono (C5) e pontuação total do PSQI. Essas alterações estão diretamente ligadas às preocupações diárias e o estresse. Já se sabe que o estresse provoca uma tensão física e psicológica no indivíduo, queda de capacidade intelectual e perturbações do sono ${ }^{21,22}$. Isso faz com que os indivíduos não consigam se desligar e descansar de fato, levando a distúrbios do sono como sonolência excessiva diurna, por exemplo. Além disso, ocorre também a procura por medicações para dormir, o que pode ser uma explicação para os presentes resultados.

Como esperado, a qualidade do sono autorrelatada no CAT, em que se questiona se o indivíduo dorme profundamente ou não, se correlacionou ao componente 5 - distúrbios do sono do PSQI $(r=0,73)$, em que é analisada a frequência com que o indivíduo acorda no meio da noite ou de manhã cedo. Dessa forma podemos salientar a importância do aprofundamento na análise da qualidade do sono em indivíduos que apresentem achados que reflitam alterações em questões singulares de instrumentos inespecíficos, como no caso o CAT. Isso se deve ao fato de que quando se tem uma avaliação em um instrumento não específico, a avaliação detalhada pode trazer informações mais claras e específicas da qualidade do sono desses indivíduos, o que facilita a proposta de uma intervenção mais eficaz.

Outro fator importante e que possui forte correlação com a qualidade do sono de indivíduos com DPOC é a dispneia, que está diretamente ligada ao tempo que demora entre o ato de deitar-se e pegar no sono de fato. Sabe-se que a privação do sono acarreta vários distúrbios e que um prejuízo na latência do sono ocasiona uma interferência no tempo total de sono e consequentemente no bem-estar físico e mental, ocasionando prejuízos em suas atividades sociais como trabalho e relações interpessoais, afetando a memória e atenção ${ }^{23}$. Levando em consideração que a dispneia é um sintoma muito comum e relatado pela grande maioria dos indivíduos com DPOC, e que apresenta piora com a progressão da doença, ressalta-se a importância de estudos que investiguem essa correlação (como o presente estudo), mas também que avaliem se tratamentos voltados diretamente para controle da dispneia refletem na qualidade do sono de indivíduos com DPOC.

Apesar do ineditismo e dos pontos fortes do estudo, algumas limitações precisam ser destacadas, como o tamanho da amostra, a ausência de indivíduos classificados como GOLD 1 e o fato de que foram utilizadas apenas avaliações subjetivas. Entretanto o presente estudo apresentou achados que foram de encontro com a literatura atual e a expandiram, e enfatizamos que levar em consideração a validade e utilidade das impressões pessoais dos participantes frente à suas queixas a respeito da doença e o que isso implica em sua qualidade do sono. Para avançar em pesquisas futuras sobre esse campo do conhecimento, podem ser realizados novos estudos com o objetivo de investigar longitudinalmente a causalidade entre a qualidade do sono e os sintomas autorrelatados por indivíduos com DPOC, além de comparar criticamente as diferentes formas de avaliação.

\section{CONCLUSÃO}

A qualidade do sono está moderadamente a fortemente correlacionada com os sintomas respiratórios, assim como com aspectos sistêmicos e emocionais comumente relatados por indivíduos com DPOC. Especificamente podemos citar a fadiga, as questões emocionais e a dispneia, além da qualidade de vida e da funcionalidade destes indivíduos. Portanto, o aprofundamento no estudo de intervenções na qualidade do sono tem o potencial de gerar benefícios em vários aspectos importantes da vida desta população.

\section{FONTE DE FINANCIAMENTO}

Nada a declarar.

\section{CONFLITO DE INTERESSES}

Nada a declarar.

\section{AGRADECIMENTOS}

Os autores do presente estudo agradecem a todos os colegas do Laboratório de Pesquisa em Fisioterapia Pulmonar (LFIP) pela contribuição no estudo.

\section{REFERÊNCIAS}

1. Global Strategy for the Diagnosis. Management and Prevention of COPD, Global Initiative for Chronic Obstructive Lung Disease (GOLD) 2020 [Internet]. Bethesda: GOLD; 2019 [citado em 2020 Set 2]. Available from: https://goldcopd. org/wp-content/uploads/2019/11/GOLD-2020-REPORTver1.0wms.pdf. Acesso em setembro de 2020.

2. Oga $T$, Nishimura $K$, Tsukino M, Sato $S$, Hajiro T. Analysis of the factors related to mortality in chronic obstructive pulmonary disease: role of exercise capacity and health status. Am J Respir Crit Care Med. 2003;167(4):544-9. http:// dx.doi.org/10.1164/rccm.200206-5830C. PMid:12446268.

3. Pitta F, Troosters T, Spruit MA, Probst VS, Decramer M, Gosselink R. Characteristics of physical activities in daily life in chronic obstructive pulmonary disease. Am J Respir Crit Care Med. 2005;171(9):972-7. http://dx.doi.org/10.1164/ rccm.200407-8550C. PMid:15665324.

4. Loivos LP. DPOC: definições e conceitos - bases clínicas. Rev Atualizações Temáticas. 2009;1(1):34-7. 
5. Shorofsky M, Bourbeau J, Kimoff J, Jen R, Malhotra A, Ayas N, et al. Impaired Sleep Quality in COPD is associated with exacerbations: The CanCOLD Cohort Study. Chest. 2019;156(5):852-63. http://dx.doi.org/10.1016/j. chest.2019.04.132. PMid:31150638.

6. Nunes DM, Mota RM, Pontes OL No, Pereira ED, Bruin VM, Bruin PF. Impaired sleep reduces quality of life in chronic obstructive pulmonary disease. Lung. 2009;187(3):15963. http://dx.doi.org/10.1007/s00408-009-9147-5. PMid:19399553.

7. Miller MR, Hankinson J, Brusasco V, Burgos F, Casaburi R, Coates A, et al. Standardisation of spirometry. Eur Respir J. 2005;26(2):319-38. http://dx.doi.org/10.1183/09031936.05 .00034805. PMid:16055882.

8. Pereira CAC, Sato T, Rodrigues SC. New reference values for forced spirometry in white adults in Brazil. J Bras Pneumol. 2007;33(4):397-406. http://dx.doi.org/10.1590/S180637132007000400008. PMid:17982531.

9. Buysse DJ, Reynolds CF 3rd, Monk TH, Berman SR, Kupfer DJ. The Pittsburgh Sleep Quality Index: a new instrument for psychiatric practice and research. Psychiatry Res. 1989;28(2):193-213. http://dx.doi.org/10.1016/01651781(89)90047-4. PMid:2748771.

10. Bertolazi AN, Fagondes SC, Hoff LS, Dartora EG, Miozzo IC, de Barba ME, et al. Validation of the Brazilian Portuguese version of the Pittsburgh Sleep Quality Index. Sleep Med. 2011;12(1):70-5. http://dx.doi.org/10.1016/j. sleep.2010.04.020. PMid:21145786.

11. Botega NJ, Bio MR, Zomignani MA, Garcia C Jr, Pereira WAB. Transtornos do humor em enfermaria de clínica médica e validação de escala de medida (HAD) de ansiedade e depressão. Rev Saude Publica. 1995;29(5):355-63. http://dx.doi.org/10.1590/S0034-89101995000500004. PMid:8731275.

12. Zigmond AS, Snaith RP. The hospital anxiety and depression scale. Acta Psychiatr Scand. 1983;67(6):361-70. http://dx.doi. org/10.1111/j.1600-0447.1983.tb09716.x. PMid:6880820.

13. Carpes MF, Mayer AF, Simon KM, Jardim JR, Garrod R. The Brazilian Portuguese version of the London Chest Activity of Daily Living scale for use in patients with chronic obstructive pulmonary disease. J Bras Pneumol. 2008;34(3):143-51. http://dx.doi.org/10.1590/S1806-37132008000300004. PMid:18392462.

14. Pitta F, Probst VS, Kovelis D, Segretti NO, Leoni AMT, Garrod R, et al. Validation of the Portuguese version of the London Chest Activity of Daily Living Scale (LCADL) in chronic obstructive pulmonary disease patients. Rev Port Pneumol. 2008;14(1):27-47. http://dx.doi.org/10.1016/ S0873-2159(15)30217-8. PMid:18265916.

15. Kovelis D, Segretti NO, Probst VS, Lareau SC, Brunetto AF, Pitta F. Validation of the modified pulmonary functional status and dyspnea questionnaire and the medical research council scale for use in Brazilian patients with chronic obstructive pulmonary disease. J Bras Pneumol. 2008;34(12):1008-18. http://dx.doi.org/10.1590/S180637132008001200005 . PMid:19180335.

16. Moreira GL, Pitta F, Ramos D, Nascimento CSC, Barzon D, Kovelis D, et al. Versão em português do Chronic Respiratory Questionnaire: estudo da validade e reprodutibilidade. J Bras Pneumol. 2009;35(8):737-44. http://dx.doi.org/10.1590/ S1806-37132009000800004. PMid:19750325.

17. Silva GPF, Morano MTAP, Viana CMS, Magalhães CBA, Pereira EDB. Portuguese-language version of the COPD Assessment Test: validation for use in Brazil. J Bras Pneumol. 2013;39(4):402-8. http://dx.doi.org/10.1590/ S1806-37132013000400002. PMid:24068260.

18. Callegari-Jacques SM. Bioestatística: princípios e aplicações. Porto Alegre: Artmed Editora; 2009.

19. Oliveira CBT. Estudo comparativo da qualidade do sono, sonolência diurna, dispneia e fadiga em pacientes com doença pulmonar obstrutiva crônica com e sem apneia obstrutiva do sono [dissertação]. Faculdade de Medicina, Universidade Federal do Ceará, Fortaleza; 2014.

20. Lal C, Kumbhare S, Strange C. Prevalence of self-reported sleep problems amongst adults with obstructive airway disease in the NHANES cohort in the United States. Sleep Breath. 2020;24(3):985-93. http://dx.doi.org/10.1007/ s11325-019-01941-0. PMid:31520299.

21. Fortes-Burgos G, Neri LA, Cupertino BFPA. Eventos estressantes, estratégias de enfrentamento, auto-eficácia e sintomas depressivos entre idosos residentes na comunidade. Psicol Reflex Crit. 2008;21(1):74-82. http:// dx.doi.org/10.1590/S0102-79722008000100010.

22. Filgueiras CJ, Hippert SIM. A polêmica em torno do conceito estresse. Psicologia (Cons Fed Psicol). 1999;19(3):40-51. http://dx.doi.org/10.1590/S1414-98931999000300005.

23. Neves G, Macedo P, Gomes M. Transtornos do sono: atualização (1/2). Rev Bras Neurol. 2017;53(3):19-30. 